Análisis. Revista de investigación filosófica, Vol. 1, nº 1, 2014, pp. 213-222

\title{
UNA NUEVA VISITA AL MUNDO DE LA VIDA
}

\author{
Fernando García Mendívil
}

Hans Blumenberg, Teoría del mundo de la vida, México: Fondo de Cultura Económica, 2013

Como señalan los editores del volumen, Hans Blumenberg no escribió ningún libro titulado Teorías del mundo de la vida, pero en su legado se encontraron cinco manuscritos clasificados bajo ese rótulo. Pese a desconocer si Blumenberg pensaba escribir un libro con ese título y, en caso afirmativo, cómo ingresarían los manuscritos en él, los editores han considerado justificada la publicación de estos textos póstumos, que se ven acompañados de dos textos de temática similar publicados en vida de Blumenberg.

Los editores no pretenden que ninguno de esos textos represente un desarrollo terminado del tema, pero sí consideran — con buen criterio- que el conjunto de los siete textos ofrece una argumentación suficientemente sólida como para merecer su publicación.

Esta argumentación está planteada, en gran medida, como un comentario a la obra de Husserl La crisis de las ciencias europeas y la fenomenología trascendental, con cuyas tesis presupone Blumenberg cierta familiaridad. Tratemos de exponer los hilos fundamentales de esta argumentación.

\section{EXPOSICIÓN DEL CONCEPTO DE MUNDO DE LA VIDA}

La primera tarea de Blumenberg es esbozar adecuadamente el concepto de mundo de la vida (Lebenswelt). Husserl designa con esta expresión la actitud preteórica, "ingenua", tal y como el mundo es vivido antes de ser ceñido dentro del ropaje de ideas físico-matemáticas de la ciencia natural, ropaje que convierte al mundo en algo más comprensible pero, también, más contraintuitivo. Blumenberg acepta esta caracterización negativa del mundo como negación de la actitud teórica, pero trata de ofrecer algunos de los principales rasgos positivos de este concepto:

El mundo de la vida es «una esfera donde está ausente el desconcierto» (p. 17) o, en palabras de Husserl, «el universo de lo sobreentendido» (p. 87). Se trata de un universo en el que no hay teoría porque no hay objetos para la 
teoría, es decir, porque todos ellos se entienden por sí mismos; en consecuencia, no es necesaria ninguna pretensión de entender nada (cfr. p. 87). No quiere esto decir que no haya descubrimientos en este mundo de la vida, pero sí que ninguno de ellos es producto de una actitud que busca enriquecer el bagaje de lo sabido; todos ellos son «una ocurrencia sagaz» (p. 88). Un buen ejemplo de que la mera acumulación de experiencia no destruye el mundo de la vida es el avance de la técnica a lo largo de toda la Edad Media, que no fue vivido por los contemporáneos como un progreso (cfr. 109). Es, de hecho, un error de la Edad Moderna el considerar retrospectivamente la mal llamada «aplicación» (p. 114) de las ciencias como algo posterior a la actitud teórica o a las ciencias puras, pues no sólo la primera es históricamente anterior, sino que también es autónoma.

Dado que actuar requiere ciertas predicciones, éstas no están ausentes del mundo de la vida, pero lo están de un modo distinto a las hipótesis falsables que utiliza la actitud científica. En efecto, «el mundo de la vida siempre contiene también reglas para el no funcionamiento de sus reglas» ( $p$. 113).

Un segundo término que utiliza Blumenberg es el de «premodalidad» (p. 115) o «validez premodal» (p. 134). El mundo de la vida es el mundo en el que no cabe imaginar que las cosas podrían ser de otra manera (cfr. p. 179). Pero incluso esta formulación traiciona lo que se quiere expresar, pues la idea del no poder ser de otra manera presupone la de poder ser de otra manera. Más exactamente, el mundo de la vida es la ausencia total del componente modal de la experiencia.

Una tercera forma de caracterizar el mundo de la vida es mediante la ausencia de fundamentación, lo cual es algo muy distinto de «la fundamentación negada del tipo de las respuestas teológicas a la pregunta de por qué Dios hace, permite u omite algo: "porque así lo quiere"» (p. 98). Decir que la voluntad de Dios es infundada presupone el concepto de fundamentación; el mundo de la vida es el ámbito en el que ninguno de los dos polos (la fundamentación o su negación) tienen sentido. El mundo de la vida es el lugar de los prejuicios; no debemos entender este término como una creencia necesariamente falsa, sino como una creencia que no requiere fundamentación.

El mundo de la vida se abandona en la medida en que se abandona esta ausencia de fundamentación. El abandono puede ser, por supuesto, sólo parcial. En la medida en que queden vestigios de la aceptación de que hay 
cosas que no requieren fundamentación, estaremos hablando de una supervivencia del mundo de la vida.

Así, Blumenberg cifra en la pregunta de Leibniz: «por qué es que hay algo y no más bien nada» (p. 90, cfr. 69) el abandono máximo del mundo de la vida y de lo sobreentendido. La actitud teórica consiste, en efecto, en no dar nada por supuesto, incluso si eso significa preguntar por razones que no se van a obtener. Es, en efecto, característico de la actitud teórica el filosofema del "Ignorabimus" (cfr. 102), es decir, la aparición de interrogantes cuya falta de respuesta no es contingente, sino inexorable, pese a lo cual no deja de tener sentido formular la pregunta. Por el contrario, lo sobreentendido «constituye una sanción protectora que desautoriza como un absurdo y un acto de curiosidad toda pregunta que penetre en ese ámbito» (p. 216).

Además de estas caracterizaciones, Blumenberg recoge la idea, ya presente en Husserl, aunque de forma menos clara, de que el mundo de la vida y el mundo cotidiano no son sinónimos: el mundo cotidiano es, desde luego, un mundo de la vida, pero no todo mundo de la vida es el cotidiano. En efecto, el mundo de la vida no se caracteriza por estar "antes" de la actitud teórica, sino por su carácter premodal; en esa medida, los resultados científicos procedentes de la actitud teórica pueden ser reintegrados dentro del mundo de la vida si se les priva de modalidad (es decir, del hecho de que son enunciados posibles, hipotéticos, falsables, etc.) y se los transforma en "afirmaciones premodales". En efecto, Blumenberg llama institución a «cualquier creación que, con independencia de los recursos que utilice, apunta a establecer o restablecer regulaciones de vida inmediatas, no sujetas a reflexión» (p. 77). La institucionalización se produce en todos los ámbitos, incluso el académico: la formación de escuelas no es sino una formación de comunidades con sobreentendidos compartidos (cf. p. 62-63).

En consecuencia, el mundo de la vida no tiene necesariamente que ver con contenidos cotidianos, sino que es sólo la forma en la que se enuncian sus juicios lo que determina su constitución. La teoría del mundo de la vida no investiga «los contenidos y las diferenciaciones específicas» (p. 111), sino la forma del mundo de la vida. Y esta forma no es otra que la de una totalidad de prejuicios (o sobreentendidos) (p. 133-134).

Con esto llegamos a una de las tesis principales del libro: el mundo de la vida no es algo que se abandone de una vez para siempre, sino que es una totalidad que permite dar un sentido a la vida, y en la medida en que la vida tenga un sentido, será porque está presupuesta esta totalidad. Frente a ella, la 
actitud científica, por su propia constitución, especifica y fragmenta la experiencia en una miríada de ámbitos autónomos.

Es por ello que el mundo de la vida, que se ve destruido por el avance de las ciencias, renace a su vez tras ellas: unificando de nuevo los resultados de éstas en un todo unificado. Esta «formación retrógrada de lo sobreentendido» (p. 36) que ocurre «mediante cada uno de sus logros consolidados y contra el sentido inmanente de esos logros» (p. 36) es, a ojos de Blumenberg, un sinónimo de la tecnificación, es decir, la transformación de resultados científicos en hechos del mundo cuyo funcionamiento se ignora pero cuya presencia pasa a darse por sentada. «La esfera en la que todavía no planteamos preguntas se vuelve idéntica a aquella en la que ya no planteamos más preguntas» (p. 230).

Las ciencias - y el propio Husserl, señala Blumenberg - participan de la esperanza de un «trabajo infinito» (p. 35) de transformación de la totalidad de lo sobreentendido por la totalidad de lo perfectamente inteligible. Blumenberg ve en esa esperanza un error: la tecnificación no es una "corrupción" de esta pureza de las ciencias que nos aparta sólo contingentemente de esa totalidad de intelección, sino una consecuencia inevitable. Pues lo cierto es que la fundamentación jamás nos da totalidades de sentido; sólo lo sobreentendido lo hace.

Parece difícil rebatir la tesis de que la tarea científica de la fundamentación no puede producir una totalidad de sentido, pero hay un punto que Blumenberg parece dejar sin discutir. En efecto, ¿es realmente necesaria esta formación de totalidades de sentido? Si fuera posible vivir en la incertidumbre y fragmentariedad de los resultados parciales de las ciencias, la tecnificación sería quizá sociológicamente probable, pero no filosóficamente apodíctica (que es lo que argumenta Blumenberg, para quien la tecnificación es consecuencia conceptual de la mera actitud teórica). Blumenberg considera erróneo el proceder cartesiano de abandonar todos los prejuicios de una sola vez, pues los prejuicios no son una suma de errores, sino esta totalidad de la que venimos hablando. Pero cla duda metódica es imposible, como afirma Blumenberg, o simplemente difícil? ¿Hay realmente una imposibilidad insoslayable en la tarea de no dar de antemano nada como verdadero? Blumenberg se esfuerza en ofrecer razones a favor de esta tesis, que deberán ser juzgadas por el lector.

Por supuesto, esta crítica representa un problema menor, pues incluso si el mundo de la vida es potencialmente abandonable, Blumenberg sigue 
teniendo razón en que «el prejuicio es la situación de partida por excelencia que hay que suponer como anterior a toda historia» (p. 99).

\section{EL MUNDO DE LA VIDA EN LA FENOMENOLOGÍA DE HUSSERL}

Aunque Blumenberg prosigue bastante más la tarea de clarificar el concepto de mundo de la vida, lo que hemos trazado hasta aquí ha de bastarnos para abordar el segundo problema del libro: la justificación del concepto. Pues aunque lo anterior haya podido convencernos de la coherencia interna del concepto, eso no basta para alejar la sospecha de que se trata de un mero ens rationis: ¿cómo es posible teorizar sobre un objeto que, por definición, es opuesto a la teoría? Blumenberg es consciente de éste y otros problemas del concepto de mundo de la vida, por lo que se encarga de justificar la necesidad y lugar sistemático de este concepto dentro de la fenomenología.

La fenomenología de Husserl, como «ciencia autodeclarada de las trivialidades» (p. 32), se propone «mostrar lo que cualquiera ya conoce o cree conocer» (p. 32). El método adecuado para ello es la reducción eidética, es decir, la progresiva eliminación de caracteres secundarios que acaba dejando como resto la esencia de algo. La confusión de los caracteres secundarios con la esencia de algo es producto de un prejuicio que, por sobreentendido, no reexamina sus fundamentos. En esa medida, la fenomenología entiende, como tarea de la filosofía, la tematización de todos los prejuicios (se entiende: de los prejuicios fundamentales del conocimiento, no de cualesquiera prejuicios empíricos de cada cual).

Frente a esta concepción de la filosofía, el neokantismo contemporáneo entiende, por contra, que la única tarea de la filosofía es examinar las condiciones de posibilidad de la ciencia. Ésta se toma como el hecho fundamental de la existencia humana, de tal manera que preguntar por el fundamento de la ciencia implica no comprender los límites del conocimiento humano: ese género de preguntas pertenece a la metafísica precritica.

Husserl señala, sin embargo, que la ciencia es contingente, en la medida en que su ausencia es perfectamente pensable: «la ciencia no es el hecho que se asegura a sí mismo» (p. 30). Por tanto, la concepción neokantiana de la ciencia, que deja sin tematizar su posible contingencia, es precisamente uno de esos prejuicios necesitados de una reducción eidética. En otras palabras: Husserl no duda de que la filosofía deba exponer las condiciones de posibilidad de la 
ciencia, pero antes y sobre todo debe aclarar si a la esencia de la ciencia le corresponde esa presunta ineludibilidad, o si más bien hay que declarar la vocación de la humanidad hacia la ciencia como una mera contingencia histórica.

El camino correcto, a juicio de Husserl, es la primera opción: la ciencia no es contingente, pues la filosofía es capaz de fundamentar su necesidad. De lo que se trata, entonces, es de buscarle a la ciencia el "ssuelo vital" de sus posiciones, movimientos y construcciones» (p. 28). Es decir: el estudio fenomenológico de la esencia de la ciencia no dará por sentada su existencia, sino que hallará los fundamentos de ésta en ese suelo vital precientífico cuyo nombre ya conocemos: el mundo de la vida.

Con esto se descarta la sospecha de que el mundo de la vida sea un ens rationis: muy al contrario, es un concepto que no nos inventamos, sino que nos surge justificadamente en la tarea de solventar el prejuicio que concierne al carácter sobreentendido de la ciencia.

Pero, pese a justificarse con esto su lugar sistemático en la filosofía, sigue quedando sin resolver el obstáculo que nombramos al principio. En efecto, tener un concepto del mundo de la vida nos permitiría conocer algo que, por definición, es previo al conocimiento. Esta contradicción, difícilmente resoluble, es soslayada por Blumenberg, al argumentar que el mundo de la vida no proviene de una reducción eidética (cfr. 11) y, en esa medida, no es un concepto en sentido estricto. Por contra, el mundo de la vida es un concepto límite, que se define «no como un mundo existente y menos aún existente por separado, sino como el concepto límite de la tendencia a lo sobreentendido» ( $\mathrm{p}$. 72). Del mismo modo que en la filosofía de Kant, un concepto límite no nos permite conocer un objeto, sino que expresa la ineludibilidad de un problema.

Una vez aclarado el estatuto del mundo de la vida como concepto límite, la tarea aparentemente sencilla de aclarar un prejuicio (el de la ineludibilidad de la ciencia) se revela, en realidad, como la tarea fundamental de aclarar todos los prejuicios, pues el concepto de la vida se define, en tanto que concepto límite, como suma y —más importante- totalidad de todos los prejuicios. Lo que expresa este concepto es el problema de que a las investigaciones particulares de cada uno de los prejuicios les subyace el problema de que éstos forman una totalidad, y de que eliminarlos uno a uno mediante la reducción eidética quizá no sea factible. En esta medida, el concepto de mundo de la vida, surgido de lo que aparentaba ser una reducción eidética particular, se revela como el problema singular que promete trazar el 
límite de las capacidades de este instrumento fenomenológico.

\section{El ARGUMENTO TRASCENDENTAL DEL MUNDO DE LA VIDA}

Una vez aclarado todo lo anterior, resulta claro cuál es la tarea que Husserl debe realizar. Es necesario «retrotraerse a una esfera en la que la vida están tan sobreentendida que es en ella donde pueden aparecer primero vivencias antepredicativas que fundamentan operaciones lógicas posteriores» (p. 79).

$\mathrm{Y}$ aquí es donde Blumenberg nos anuncia que, en realidad, Husserl fracasó en su propósito. Veamos en detalle el argumento de Husserl y la crítica de Blumenberg:

Husserl, pese a concebir el mundo de la vida como previo a toda actitud teórica, «toma este comienzo de la historia de toda teoría como el resultado de un acto de voluntad que no es necesario indagar» (p. 82). Y no le faltan argumentos: «se declara contradictorio proporcionarle un comienzo a la historia y a la vez plantear la pregunta por lo que había antes de ese comienzo y por cómo se llegó a ese comienzo, pues eso ya sería un trozo de historia» (p. $85)$.

El argumento parece sólido; sin embargo, es aquí donde Blumenberg acusa a Husserl del inesperado delito de neokantismo, que se manifiesta en «la disposición a no seguir indagando el hecho primigenio y a tomarlo como mera normalización de todo lo sucesivo» (p. 83). En efecto, a ojos de Blumenberg, desarrollar todo el aparato conceptual del mundo de la vida para terminar diciendo que el comienzo de la actitud teórica es un acto de voluntad insondable no es otra cosa que dar un largo rodeo para llegar a la posición neokantiana que se quería evitar. Obviamente, la posición neokantiana y la de Husserl no son exactamente la misma: Husserl investiga la fundamentación de la ciencia y llega a que es insondable, mientras que los neokantianos ya partían directa -e injustificadamente- de que era insondable. Pero para Blumenberg, la diferencia es inesencial, comparado con lo que tienen en común: el admitir que, a partir de determinado punto (un poco más lejano en el caso de Husserl), carece de sentido la pregunta por la fundamentación de la ciencia.

Pero esta posición común es necesariamente un error: si la actitud teórica consiste, en definitiva, en no dejar nada sin fundamentar, ¿cómo es aceptable dejar sin fundamentar esta propia actitud teórica? Como dice Blumenberg, la actitud teórica «debe tener que empezar para que no sea o no 
pueda volverse un mero fósil cultural» (p. 131). En efecto, la actitud teórica, que desestima todo aquello que no tenga otra razón de ser que la de ser un producto cultural de la sociedad en la que habita, necesariamente ha de desestimarse a sí misma si no consigue una fundamentación y deja su origen como mero hecho contingente de la historia de Europa, tal y como hacen tanto la filosofía neokantiana como Husserl.

Frente a esto, Blumenberg se propone dar una verdadera fundamentación; aquella que Husserl buscó o debería haber buscado. Veamos cómo:

Blumenberg no niega la correción del argumento de Husserl que acabamos de indicar, según el cual sería contradictorio que antes de la historia hubiera historia. Pero esto no nos exime de buscar una justificación; lo único que hace es obligarnos a buscar una solución diferente. Y Blumenberg posee una: el mundo de la vida no es una etapa previa a la historia de la teoría; sino un «concepto trascendental» (p. 86).

$\mathrm{El}$ argumento trascendental que Blumenberg propone ya no habla del paso de una época histórica a otra, sino del paso entre dos ámbitos conceptuales. El primero es el mundo de la vida, donde «la carga probatoria recae siempre en quien exige fundamentación» (p. 260); el segundo, la actitud teórica o «comportamiento en el que se demanda y se concede fundamentación sin que sea necesario fundamentar esa demanda y esa concesión en cuanto tales» (p. 260-261).

Partiendo del mundo de la vida, «tiene que ser posible demostrar que ese estatus no puede perdurar por sí mismo y que su inestabilidad aparece como [...] punto de partida para la teoría» (p. 60). Es decir, el proyecto de Blumenberg es partir del mundo de la vida, de la ausencia total de actitud teórica, y demostrar la inevitable contradicción interna y por ende "derrumbamiento" de este mundo, "derrumbamiento" que da lugar a la actitud teórica. Llevando a cabo esta argumentación, la actitud teórica no se dejará ni como contingencia, ni como factum insondable, sino que quedará fundamentada por la contradicción interna de su ausencia.

En palabras de Blumenberg, de lo que se trata es de «imaginarnos las condiciones extremas de imposibilidad de la filosofía, para hacer que la filosofía se vuelva posible a partir de esas mismas condiciones» (p. 131-132). «La filosofía no puede ser pensada naciendo de la no filosofía, sino surgiendo de la imposibilidad de la filosofía» (p. 143). La diferencia es sutil, pero importante: si la filosofía nace de la no filosofía, es un hecho histórico, 
contingente; si nace de la imposibilidad de la filosofía nos encontramos ante una fundamentación trascendental. En efecto, si partiendo de una conciencia abierta a un mundo, pero carente de actitud teórica, se demuestra que esta situación es conceptualmente contradictoria, lo que se demostrará es que un mundo abierto a una conciencia es necesariamente accesible por medio de la actitud teórica, y que la actitud teórica no es una propiedad secundaria "opcional", sino una propiedad esencial del binomio conciencia-mundo. En otras palabras, si la ausencia de actitud teórica es incoherente, lo que se demuestra con ello es que la presencia de esa actitud teórica no es un hecho dentro de la experiencia de esa conciencia, sino, por contra, una condición de posibilidad de que haya, en general, algo así como experiencia. Que Husserl no viera esto fue la «insuficiencia radical de su fenomenología del mundo de la vida» (p. 143).

El modelo de argumentación de Blumenberg no es novedoso, y él mismo señala varias veces que es el mismo que el de Hobbes y su status naturalis. Se trata, en efecto, de un estado que no ha existido nunca, pero cuya contradicción interna fundamenta el surgimiento del soberano (es decir, de la ley). El estado de naturaleza en Hobbes «produce forzosamente desde sí lo que ya no es él mismo» (p. 45).

\section{CONCLUSIÓN}

Lo que resta es la tarea de culminar esta argumentación de que el mundo de la vida es internamente contradictorio. Blumenberg dedica el resto del libro a ofrecer esta argumentación. Es, sin embargo, discutible que esta argumentación sea la «historia trascendental» (p. 87) que Blumenberg pretende y que no se haya visto contaminada por la historia fáctica. Así, por ejemplo, el excesivo hincapié en la «inestabilidad» (p. 114) de la vida, que termina acarreando un problema para el mundo de la vida, parece desatender el hecho de que la posibilidad alternativa de una estabilidad ininterrumpida en las leyes de la naturaleza no es, en principio, contradictoria; en consecuencia, difícilmente podrá ser una argumentación trascendental si consta de un hecho contingente. Igualmente fuera de lugar resultan los excursos sobre la bipedestación de los homínidos, en la medida en que lo que Blumenberg pretende esclarecer es la fundamentación trascendental de la conciencia teórica, y no el surgimiento de la función cerebral en el Homo sapiens; este segundo interrogante es un investigación biológica o histórica independiente de la 
primera ( $\mathrm{o}$, más precisamente, una investigación empírica que presupone la investigación trascendental que Blumenberg se había propuesto).

Será el lector quien juzgue el triunfo o fracaso de la pretendida argumentación trascendental. Sin embargo, su éxito no es lo único importante. Como escribe el propio Blumenberg: «No es imprescindible que una teoría cumpla con las exigencias de carga probatoria que se autoimpone para ser eficaz ante aquellas que se han quedado aún más atrás de tales exigencias» (p. 140). Incluso si Blumenberg no triunfa en su tarea, lo que es difícil rebatirle es la inevitabilidad de tal argumentación para todo aquel que quiera seguir investigando el mundo de la vida: si Blumenberg no lo ha conseguido, la tarea sigue pendiente. 